\title{
Zur Integration von Erneuerbaren Energien und der dezentralen Kraft-Wärme-Kopplung in die Elektrizitätsversorgung
}

\author{
Wolf Fichtner
}

Online veröffentlicht: 17.11.2009

(C) Springer-Verlag 2009

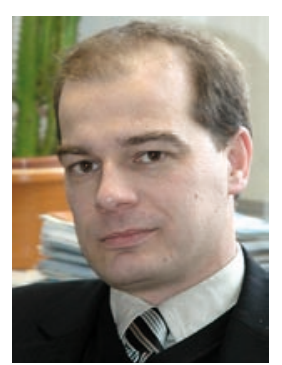

Die Energiewirtschaft befindet sich derzeit in einem tief greifenden Wandel. Die vielfältigen, teilweise konträren Anforderungen hinsichtlich Klimaschutz, Versorgungssicherheit und Wirtschaftlichkeit verdeutlichen, dass die Sicherung einer - diese Kriterien erfüllenden - nachhaltig(er)en Energieversorgung zu den größten globalen Herausforderungen der Zukunft gehört. Daher ist es wenig erstaunlich, dass sich die energiewirtschaftlichen Rahmenbedingungen in den vergangenen Jahren bereits stark verändert haben und sich auch in den nächsten Jahren weiter verändern werden. So verpflichtete sich bspw. die EU dazu, die Treibhausgasemissionen bis 2020 um mindestens $20 \%$, bei vergleichbaren Anstrengungen in anderen (Industrie-)Ländern sogar um 30 \% gegenüber den Emissionen des Basisjahrs 1990 zu reduzieren. Unter anderem um diese Klimaziele zu erreichen, soll der Endenergiebedarf bis 2020 zu $20 \%$ aus Erneuerbaren Energien gedeckt werden. Die Bundesregierung hat sich ihrerseits die Ziele gesetzt, bis 2020 den Anteil Erneuerbarer

Prof. Dr. Wolf Fichtner $(\bowtie)$

Karlsruher Institut für Technologie (KIT),

Institut für Industriebetriebslehre und Industrielle Produktion (IIP), Lehrstuhl für Energiewirtschaft,

76187 Karlsruhe, Deutschland

E-Mail: wolf.fichtner@kit.de
Energien an der Stromversorgung auf mindestens $30 \% \mathrm{zu}$ erhöhen und den Anteil der Strombereitstellung aus KraftWärme-Kopplungsanlagen auf etwa $25 \%$ zu verdoppeln.

Bereits diese Zahlen verdeutlichen, dass die Energiebereitstellung aus dezentralen Anlagen auf Basis Erneuerbarer Energien und der Kraft-Wärme-Kopplung (KWK) in den kommenden Jahren bedeutende Anteile erreichen wird. Der zukünftige Elektrizitätsmix in Deutschland wird daher durch ein Miteinander dezentraler wie auch - weiterhin notwendiger - zentraler Bereitstellungsanlagen geprägt sein. Allerdings werden Windkraftanlagen, die heutzutage dominierende erneuerbare Bereitstellungsoption, aufgrund der besseren Windverhältnisse vor allem in Norddeutschland errichtet, was zu neuen Herausforderungen für den Transport von elektrischer Energie in Richtung Süden führt, da die bestehenden Netze hierfür nur bedingt ausgelegt sind und es daher zu Netzengpässen kommen kann. Entstehen in den Elektrizitätsnetzen solche Engpässe, scheinen langfristig Netzausbaumaßnahmen unausweichlich, denen allerdings oftmals Hemmnisse, bspw. in Form von lokalen Widerständen, entgegen stehen. Innerhalb Deutschlands verhindern die Übertragungsnetzbetreiber gegenwärtig kurzfristig mögliche Engpässe mit Hilfe des kostenbasierten Redispatch, wobei (in der Regel konventionelle) Kraftwerksleistung in der Region vor dem Engpass reduziert und Leistung hinter dem Engpass erhöht wird. Damit kann die Teilung des deutschen Strommarkts in verschiedene Preiszonen verhindert und ein einheitlicher Strommarkt gewährleistet werden. Grenzüberschreitende Übertragungskapazitäten werden bei Knappheit den Marktteilnehmern nach nichtdiskriminierenden, marktorientierten Methoden zur Verfügung gestellt.

Des Weiteren kann die Elektrizitätseinspeisung aus Anlagen auf Basis Erneuerbarer Energien und der KWK vor allem in Schwachlastzeiten zu einem Leistungsüberschuss führen. Um künftig die dargebotene (regenerative) Ener- 
gie dennoch möglichst vollständig nutzen zu können (und möglichst wenig auf das sogenannte Erzeugungsmanagement, d.h. die Anpassung der Erzeugung an den Bedarf, zurückgreifen zu müssen), bedarf es intelligenter Netze, neuer Speichertechnologien und Nachfrager, deren Bedarf an die Bereitstellung angepasst werden kann. Hier eröffnen sich Potenziale durch das Verschieben von Lasten bspw. für Kühlzwecke bei mit intelligenten Zählern ausgestatteten Kunden, um so den Ausgleich zwischen Verbrauch und (zunehmend fluktuierender) Bereitstellung sicherzustellen. Große Hoffnungen werden in diesem Zusammenhang auf neue Elektrizitätsanwendungen gesetzt, wie etwa in die durch technische Fortschritte insbesondere bei LithiumIonen Batterien momentan stark propagierte Elektromobilität. So sollen Erneuerbare Energien durch gezieltes Be- und Entladen von Elektroautos besser in das Elektrizitätssystem integriert werden. Abschließend ist zu betonen, dass zur Realisierung eines solchen intelligenten Elektrizitätssystems vor allem auch eine an die technische EnergieInfrastruktur angekoppelte leistungsfähige und rechtskonforme Informations- und Kommunikationsinfrastruktur benötigt wird.

Im Rahmen der vorliegenden uwf-Ausgabe sollen verschiedene Aspekte der Integration von Erneuerbaren Energien und der dezentralen Kraft-Wärme-Kopplung in die Elektrizitätsversorgung aufgezeigt werden, um so das skizzierte Problemfeld möglichst umfassend beleuchten zu können. Nach der Darstellung der zukünftigen Entwicklung Erneuerbarer Energien in Deutschland (Genoese, F. et al.: „Zukünftige Entwicklung von Erneuerbaren Energien in Deutschland und Anforderungen an das Energiesystem") und der dezentralen Bereitstellung durch Mikro-KWK-Anlagen (von Roon, S. et al.: „Dezentrale Bereitstellung von Strom und Wärme mit Mikro-KWK-Anlagen“) liegt ein weiterer Schwerpunkt auf der Schnittstelle zwischen Erzeu- gung und Nachfrage: den Netzen und der Behandlung von Netzengpässen im europäischen (Spiecker, S. et al.: „Ökonomische Bewertung von Netzengpässen und Netzinvestitionen“) und deutschen (Borggrefe, F. et al.: „Auswirkungen fluktuierender Windverstromung auf Strommärkte und Übertragungsnetze") Transportnetz sowie auf untergelagerten Netzen (Pfeiffer, K. et al.: „Netzengpässe in Verteilnetzen und technische Lösungsmöglichkeiten"). Anschließend werden Möglichkeiten zur Energiespeicherung aufgezeigt (Dötsch, C. et al.: „Speicherung elektrischer Energie“), bevor verschiedene Optionen zur Anpassung des Bedarfs an die Erzeugung vorgestellt werden. Hierzu widmet sich ein Beitrag einem Energiemanagementsystem, mit dessen Hilfe Geräte wie Kühlschränke oder Waschmaschinen durch Preissignale gesteuert werden (Nestle, D. et al.: „Integration dezentraler und erneuerbarer Energien durch variable Strompreise im liberalisierten Energiemarkt"). Direkt mit einer solchen Idee des Smart Home verbunden sind die Ausführungen zur Nutzung von Wärme und Kälte in Gebäuden (Schmidt, F.: „Mögliche Beiträge der Gebäudeheizung und -kühlung zum effizienten Betrieb intelligenter Stromnetze"), was vor dem Hintergrund, dass noch immer der größte Anteil des Energiebedarfs von Gebäuden durch die Wärmenutzung verursacht wird, von besonderer Bedeutung ist. Abgeschlossen wird diese Schwerpunktausgabe durch einen Beitrag zur verbesserten Integration Erneuerbarer Energien mit Hilfe elektrisch betriebener Fahrzeuge (Schönfelder, M. et al.: „Elektromobilität“), nicht zuletzt weil sich Deutschland zum Ziel gesetzt hat, ein Leitmarkt für Elektromobilität zu werden.

An dieser Stelle sei allen Beteiligten für ihr Engagement gedankt: den Autoren für ihre kreativen Beiträge, den Herausgebern für die Möglichkeit zu diesem Schwerpunktheft und der uwf-Redaktion für die fachkundige Unterstützung. 